\title{
The eighth edition TNM stage classification for lung cancer: What does it mean on main street?
}

\author{
Frank C. Detterbeck, MD
}

From the Division of Thoracic Surgery, Yale University, New Haven, Conn

Received for publication March 6, 2017; accepted for publication Aug 26, 2017; available ahead of print Oct 20, 2017.

Address for reprints: Frank C. Detterbeck, MD, Department of Thoracic Surgery, Yale University, 330 Cedar S, BB 205, New Haven, CT 06520-8062 (E-mail: frank.detterbeck@yale.edu).

J Thorac Cardiovasc Surg 2018;155:356-9 $0022-5223 / \$ 36.00$

Copyright (c) 2017 Published by Elsevier Inc. on behalf of The American Association for Thoracic Surgery https://doi.org/10.1016/j.jtcvs.2017.08.138

Feature Editor's Note-The eighth edition of the American Joint Commission on Cancer TNM staging system for nonsmall cell lung cancer was introduced in January 2017 and will be implemented in the United States in January 2018. The eighth edition has been bolstered with a number of data-driven updates that will substantially affect the staging of early non-small cell lung cancer and are critical for the thoracic surgical oncologist to embrace in daily practice. New entities such as TImi, TIc, MIc, and stage IAl tumors, among others, will become regular in the language that we use to care for patients with lung cancer, and this staging framework is critical for advancing the field of thoracic oncology. I therefore submit that mastery of this system is our fundamental duty. In this issue of the Journal, we are privileged to have a Featured Expert Opinion article outlining these updates, written by a long-standing and ongoing participant in the staging projects that formulate our national and international staging systems for thoracic malignancies. Herein is a succinct primer to welcome in the eighth edition.

\section{Bryan Burt, MD, Thoracic Feature Editor}

Stage classification is an essential, fundamental cornerstone to delivering care for patients with cancer. It provides a nomenclature to describe the anatomic extent of the tumor. These tumor characteristics have a major impact on prognosis as well as on the therapeutic options. It is critical that we can communicate clearly with one another, both in discussions and in evaluating whether the results of a clinical trial are applicable to a patient in front of us in the clinic, because disease extent is one of the major inclusion and exclusion criteria in most studies. It is nearly impossible to imagine delivering care to patients with cancer without being able to communicate about the tumor stage.

The fundamental structure of stage classification is the TNM system: $T$ for characteristics of the primary tumor, $N$ for nodal involvement, and $M$ for (distant) metastasis. Specific T, N, and $\mathrm{M}$ categories that exhibit similar behavior are then coalesced into stage groups. The bodies that

\begin{tabular}{|c|c|c|c|c|c|}
\hline $\mathrm{T} / \mathrm{M}$ & Subcategory & No & N1 & N2 & N3 \\
\hline \multirow[t]{3}{*}{$\mathrm{T} 1$} & Tla & IA1 & IIB & IIIA & IIIB \\
\hline & T1b & IA2 & IIB & IIIA & IIIВ \\
\hline & T1c & IA3 & IIB & IIIA & IIIB \\
\hline \multirow[t]{2}{*}{$\mathrm{T} 2$} & $\mathrm{~T} 2 \mathrm{a}$ & IB & IIB & IIIA & IIIIB \\
\hline & $\mathrm{T} 2 \mathrm{~b}$ & IIA & IIB & IIIA & IIIIB \\
\hline $\mathrm{T} 3$ & T3 & IIB & IIIA & IIIB & IIIC \\
\hline $\mathrm{T} 4$ & $\mathrm{~T} 4$ & IIIA & IIIA & IIIIB & IIIC \\
\hline \multirow[t]{3}{*}{ M1 } & M1a & IVA & IVA & IVA & IVA \\
\hline & M1b & IVA & IVA & IVA & IVA \\
\hline & M1c & IVB & IVB & IVB & IVB \\
\hline \multicolumn{6}{|c|}{ Lung cancer stage groups. } \\
\hline \multicolumn{6}{|c|}{ Central Message } \\
\hline \multicolumn{6}{|c|}{$\begin{array}{l}\text { Consistent stage classification is a fundamental } \\
\text { aspect of management of patients with cancer. } \\
\text { It is essential that clinicians be aware of the } \\
\text { new 8th edition of the TNM classification of } \\
\text { lung cancer. }\end{array}$} \\
\hline
\end{tabular}

See Editorial Commentary page 360.

See Editorial page 354.

formally decide on the definitions of the $\mathrm{T}, \mathrm{N}$, and $\mathrm{M}$ categories and stage groups are the American Joint Commission on Cancer (AJCC) in the United States and the Union for International Cancer Control (UICC) internationally. Although it is critical that stage classification represents a stable, consistently used nomenclature, periodic revisions are needed as technology changes and the ability to define nuances regarding tumor extent progresses. The AJCC and UICC therefore undertake periodic revisions, and January 1, 2017 marks the introduction of the eighth edition of the TNM classification.

Lung cancer is unique among cancer sites with regards to stage classification. Most cancer stage classification systems are largely empiric and consensus based. The lung cancer stage classification, which is developed by the International Association for the Study of Lung Cancer (IASLC), is based on a sophisticated statistical analysis of an international database of more than 100,000 patients. This analysis has been published in a series of articles in the Journal of Thoracic Oncology, specifically addressing the $\mathrm{T}, \mathrm{N}$, and $\mathrm{M}$ components; the stage groups (Table 1 and Figure 1); and the methodology and validation used for non-small cell lung cancer and small cell lung cancer. $^{2-7}$ Additional articles address details of tumor size measurement and the TNM classification of lung cancers 
TABLE 1. Definitions for TNM descriptors

T (primary tumor)

\begin{tabular}{ll}
\hline T0 & No primary tumor \\
Tis & Carcinoma in situ (squamous or adenocarcinoma) \\
T1 & Tumor $\leq 3 \mathrm{~cm}$ \\
T1mi & Minimally invasive adenocarcinoma \\
T1a & Superficial spreading tumor in central airways* \\
T1a & Tumor $\leq 1 \mathrm{~cm}$ \\
T1b & Tumor $>1$ but $\leq 2 \mathrm{~cm}$ \\
T1c & Tumor $>2$ but $\leq 3 \mathrm{~cm}$ \\
T2 & Tumor $>3$ but $\leq 5 \mathrm{~cm}$ or tumor involving: visceral pleura, $\dagger$ main bronchus (not carina), atelectasis to hilum $\dagger$ \\
T2a & Tumor $>3$ but $\leq 4 \mathrm{~cm}$ \\
T2b & Tumor $>4$ but $\leq 5 \mathrm{~cm}$ \\
T3 & Tumor $>5$ but $\leq 7 \mathrm{~cm}$ or invading chest wall, pericardium, phrenic nerve; or separate tumor nodule(s) in the same lobe \\
T4 & Tumor $>7 \mathrm{~cm}$ or tumor invading: mediastinum, diaphragm, heart, great vessels, recurrent laryngeal nerve, carina, trachea, esophagus, \\
& spine; or tumor nodule(s) in a different ipsilateral lobe \\
\hline
\end{tabular}

\begin{tabular}{ll}
\hline & \multicolumn{1}{c}{ N (regional lymph nodes) } \\
\hline N0 & No regional node metastasis \\
N1 & Metastasis in ipsilateral pulmonary or hilar nodes \\
N2 & Metastasis in ipsilateral mediastinal or subcarinal nodes \\
N3 & Metastasis in contralateral mediastinal, hilar, or supraclavicular nodes \\
\hline & \multicolumn{1}{c}{ M (distant metastasis) } \\
\hline M0 & No distant metastasis \\
M1a & Malignant pleural or pericardial effusion $\ddagger$ or pleural or pericardial nodules or separate tumor nodule(s) in a contralateral lobe \\
\hline M1b & Single extrathoracic metastasis \\
M1c & Multiple extrathoracic metastases $(1$ or $>1$ organ) \\
*Superficial spreading tumor of any size but confined to the tracheal or bronchial wall. $\dagger$ Atelectasis or obstructive pneumonitis extending to hilum; such tumors are classified as \\
T2a if $>3$ and $\leq 4 \mathrm{~cm}, \mathrm{~T} 2 \mathrm{~b}$ if $>4$ and $\leq 5 \mathrm{~cm}$. †ेPleural effusions are excluded that are cytologically negative, nonbloody, transudative, and clinically judged not to be due to cancer.
\end{tabular}

involving multiple pulmonary sites of disease (second primary cancer, additional tumor nodules, multifocal ground glass and lepidic adenocarcinomas, and pneumonic-type adenocarcinoma). ${ }^{8-12}$ Key features to highlight that distinguish the eighth from the seventh edition are summarized in Table 2.

It is essential for clinicians not only to be informed about the new stage classification system but to adopt it. The stage classification is such an essential part of how we communicate about patients with cancer that we cannot afford to be inconsistent. This applies to how we designate the tumors of our patients in our notes and medical records, as well as in publications and clinical trials. As with any transition, there is always a period of needing to learn a new system and adopt new habits. The AJCC and UICC eighth edition staging manuals are published and available for purchase. More focused on lung cancer and other thoracic malignancies is the IASLC Staging Handbook in Thoracic Oncology and the IASLC pocket cards, which can provide an at-yourfingertips resource.

Unfortunately, a further challenge to consistency has been introduced by a discrepancy between the AJCC and the UICC. Although these organizations have traditionally collaborated closely to be entirely consistent and thus ensure uniform nomenclature throughout the world, there is some deviation from this with the eighth edition. The AJCC recently decided to delay implementation of the eighth edition stage classification in the United States until January 1, 2018, whereas the UICC (applicable to the rest of the world) is proceeding with the previously scheduled implementation of the eighth edition on January 1, 2017. The AJCC decision was made in November 2016 after discussion with multiple relevant US organizations: the National Cancer Institute Surveillance, Epidemiology, and End Results Program, the Centers for Disease Control and Prevention, the College of American Pathologists, the National Comprehensive Cancer Network, the National Cancer Data Base, and the Commission on Cancer. The decision was made to ensure that the cancer care community has the necessary infrastructure in place for documenting eighth edition staging.

For institutions and clinicians outside the United States, the process is simple - they adopted the eighth edition system as of January 1, 2017, as had been planned for many years. In the United States, tumor registrars also have a clear mandate, in this case to continue using the seventh edition 


\begin{tabular}{|c|c||c|c|c|c|}
\hline T/M & Subcategory & N0 & N1 & N2 & N3 \\
\hline \hline \multirow{3}{*}{ T1 } & T1a & IA1 & IIB & IIIA & IIIB \\
\cline { 2 - 5 } & T1b & IA2 & IIB & IIIA & IIIB \\
\cline { 2 - 5 } & T1c & IA3 & IIB & IIIA & IIIB \\
\hline \multirow{2}{*}{ T2 } & T2a & IB & IIB & IIIA & IIIB \\
\cline { 2 - 6 } & T2b & IIA & IIB & IIIA & IIIB \\
\hline T3 & T3 & IIB & IIIA & IIIB & IIIC \\
\hline T4 & T4 & IIIA & IIIA & IIIB & IIIC \\
\hline M1 & M1a & IVA & IVA & IVA & IVA \\
\cline { 2 - 6 } & M1b & IVA & IVA & IVA & IVA \\
\cline { 2 - 6 } & M1c & IVB & IVB & IVB & IVB \\
\hline
\end{tabular}

FIGURE 1. Lung cancer stage grouping (eighth edition).

classification until 2018 . This potentially means that data during 2017 from the United States and the rest of the world will not be entirely compatible. The question arises as to what US institutions that were ready and poised to adopt the new classification on January 1, 2017, should do. Although the data filed by tumor registrars must be that of the seventh edition until 2018, clinicians and institutions in the United States may decide to record both, so that the data can be aligned with that of the rest of the world. If this policy is adopted, it is essential that everyone not only document a stage classification but also the edition used, for example T1bN1M0 (seventh edition)/T1cN1M0 (eighth edition).

How does the new stage classification affect clinicians caring for patients with lung cancer? The availability of a large data set promotes greater granularity, allowing us to describe more specific tumor characteristics. In turn, this provides a mechanism to define treatment outcomes for

TABLE 2. Key features distinguishing the eighth edition classification

Tumor size is determined by the largest dimension of the solid portion by computed tomography* (clinical) or the invasive portion $\dagger$ (pathologic $\ddagger$ ).

Tumor size is subdivided in $1-\mathrm{cm}$ increments up to $5 \mathrm{~cm}$.

Tumors $>5$ but $\leq 7 \mathrm{~cm}$ are classified as $\mathrm{T} 3$.

Tumors $>7 \mathrm{~cm}$ are classified as $\mathrm{T} 4$.

Tumors involving the main stem bronchus or causing lung atelectasis or obstructive pneumonitis are classified as T2 regardless of distance

from the carina or whether they cause partial or total lung atelectasis. Tumors involving the diaphragm are classified as T4.

Tumors involving a single distant (extrathoracic) site are classified as M1b.

Tumors involving multiple distant sites are classified as M1c.

*With lung window settings, 1-mm slice thickness. †If several areas of solid and invasive tumor are present, multiply the largest total dimension (including the ground glass or lepidic components) by the proportion that is solid (imaging) or invasive (histologic). ఫNevertheless, the largest total dimension (including the ground glass or lepidic components) should be additionally noted. more specific subgroups of patients. For example, it may help us define the role of adjuvant chemotherapy for varying sizes of NOM0 tumors. It is crucial to note, however that it is the outcome of patients treated in a defined way in clinical studies that defines the best treatment approach, not the name put on a particular tumor by the stage classification. The nature of a tumor, its behavior, and its response to a treatment strategy is the same no matter what name we put on it. Greater granularity only provides a tool that helps us communicate clearly.

People often point to other tumor characteristics that may affect the choice of treatment and the resultant prognosis (eg, the presence of genetic mutations) and wonder why they are not part of the stage classification. Stage classification specifically provides a description of the anatomic extent of disease, however, thus providing a tool to describe one (major) component of how to discriminate patients that are appropriately treated in a certain fashion. There are many other characteristics that also influence the choice of treatment in specific settings, such as mutations in advanced stage tumors being considered for targeted therapy, pulmonary reserve in patients with early stage disease being considered for resection, age, comorbidities, and so on.

Similarly, the anatomic burden of disease is one aspect that affects prognosis, along with many other factors such as patient characteristics (eg, age and comorbidities), tumor factors (eg, histotype and molecular characteristics), environmental factors (eg, access to care and geographic region), and treatment-related factors (eg, treatment received, quality of care, and response to treatment). For aggressive and advanced stage cancers, prognosis may be overwhelmingly determined by the anatomic tumor extent. In other settings, however, such as less aggressive or advanced tumors, prognosis is determined largely by other factors, such as general health, age, and effectiveness of the 
curative-intent therapy used. Stage classification thus is useful as a tool to define prognosis but must be considered together with multiple other factors that influence prognosis. The number of factors influencing prognosis; the complex interplay of the magnitude of impact of a factor depending on the specific setting, treatment strategy, and other factors; and the level of certainty of our knowledge quickly overwhelm the ability to fit it into a simple prognostic grouping. Development of a prognostic model is being undertaken by the IASLC. ${ }^{13}$

The prognosis of the patients used to determine the eighth edition stage classification has limited utility in estimating the outcomes of a patient being evaluated today. The ability to discriminate between groups (definition of outcomes of groups relative to one another) is certainly applicable today-discriminatory ability and demonstrated applicability of this across geographic regions, time periods, a spectrum of lung cancer types, and so on is what the stage classification is based on. The actual outcome (calibration of prognosis), however, is not. The 5-year survivals of stage groups in the database used for the eighth edition analysis represent global averages of patients with diagnoses between 1999 and 2010 who were treated in many different ways. There are differences in outcomes by region and by specific treatment approaches, and there has been marked improvement $(30 \%-50 \%)$ in outcomes over time ${ }^{3}$; the actual outcomes in the eighth edition database are therefore not appropriate to apply to new patients with diagnoses made today in any specific setting. Prognosis derived from more recent, region-specific, and treatment-specific studies provides a more accurate estimate of actual survival.

In summary, it is essential that clinicians caring for patients with lung cancer be aware of the eighth edition stage classification system. Thoracic surgeons should be familiar with the articles defining the details of this system. We must be consistent and explicit in how we communicate about patients and in clinical analyses. It is particularly important in 2017 to be clear about which system (seventh or eighth edition) one is using, depending on one's location and institutional standard. The new system sets the stage to sort out further nuances of management of patients with particular tumor characteristics.

\section{Conflict of Interest Statement}

Author has nothing to disclose with regard to commercial support.

\section{References}

1. Rami-Porta R, Bolejack V, Giroux DJ, Chansky K, Crowley J, Asamura H, et al; International Association for the Study of Lung Cancer Staging and Prognostic Factors Committee, Advisory Board Members and Participating Institutions. The IASLC Lung Cancer Staging Project: the new database to inform the eighth edition of the TNM classification of lung cancer. J Thorac Oncol. 2014;9:1618-24.

2. Asamura H, Chansky K, Crowley J, Goldstraw P, Rusch VW, Vansteenkiste JF, et al; International Association for the Study of Lung Cancer Staging and Prognostic Factors Committee, Advisory Board Members, and Participating Institu- tions. The IASLC Lung Cancer Staging project: proposals for the revision of the $\mathrm{N}$ descriptors in the forthcoming 8th edition of the TNM classification for lung cancer. J Thorac Oncol. 2015;10:1675-84.

3. Detterbeck F, Chansky K, Groome P, Bolejack V, Crowley J, Shemanski L, et al; IASLC Staging and Prognostic Factors Committee, Advisory Boards, and Participating Institutions. The IASLC Lung Cancer Staging Project: methodology and validation used in the development of proposals for revision of the stage classification of NSCLC in the forthcoming (eighth) edition of the TNM classification of lung cancer. J Thorac Oncol. 2016;11:1433-46.

4. Eberhardt W, Mitchell J, Crowley J, Kondo H, Kim YT, Turrisi A III, et al; International Association for Study of Lung Cancer Staging and Prognostic Factors Committee, Advisory Board Members, and Participating Institutions. The IASLC Lung Cancer Staging Project: proposals for the revision of the M descriptors in the forthcoming (eighth) edition of the TNM classification of lung cancer J Thorac Oncol. 2015;10:1515-22.

5. Goldstraw P, Chansky K, Crowley J, Rami-Porta R, Asamura H, Eberhardt WE, et al; International Association for the Study of Lung Cancer Staging and Prognostic Factors Committee, Advisory Boards, and Participating Institutions; International Association for the Study of Lung Cancer Staging and Prognostic Factors Committee Advisory Boards and Participating Institutions. The IASLC Lung Cancer Staging Project: proposals for the revision of the TNM stage groupings in the forthcoming (eighth) edition of the TNM classification for lung cancer. J Thorac Oncol. 2015;11:39-51.

6. Nicholson A, Chansky K, Crowley J, Beyruti R, Kubota K, Turrisi A, et al; Staging and Prognostic Factors Committee, Advisory Boards, and Participating Institutions; Staging and Prognostic Factors Committee Advisory Boards and Participating Institutions. The IASLC Lung Cancer Staging Project: proposals for the revision of the clinical and pathologic staging of small cell lung cancer in the forthcoming eighth edition of the TMN classification for lung cancer. $J$ Thorac Oncol. 2016;11:300-11.

7. Rami-Porta R, Bolejack V, Crowley J, Ball D, Kim J, Lyons G, et al; IASLC Staging and Prognostic Factors Committee, Advisory Boards and Participating Institutions. The IASLC lung cancer staging project: proposals for the revisions of the $\mathrm{T}$ descriptors in the forthcoming eighth edition of the TNM classification for lung cancer. J Thorac Oncol. 2015;10:990-1003.

8. Detterbeck F, Nicholson F, Franklin W, Marom EM, Travis WD, Girard N, et al; IASLC Staging and Prognostic Factors Committee; Advisory Boards; Multiple Pulmonary Sites Workgroup; Participating Institutions. The IASLC Lung Cancer Staging Project: summary of proposals for revisions of the classification of lung cancers with multiple pulmonary sites of involvement in the forthcoming eighth edition of the TNM classification. J Thorac Oncol. 2016;11:639-50.

9. Detterbeck F, Bolejack V, Arenberg D, Crowley J, Donington JS, Franklin WA, et al; IASLC Staging and Prognostic Factors Committee; Advisory Boards; Multiple Pulmonary Sites Workgroup; Participating Institutions. The IASLC Lung Cancer Staging Project: background data and proposals for the classification of lung cancer with separate tumor nodules in the forthcoming eighth edition of the TNM classification for lung cancer. J Thorac Oncol. 2016;11:681-92.

10. Detterbeck F, Arenberg D, Asamura H, Girard N, Arenberg DA, Travis WD, et al; IASLC Staging and Prognostic Factors Committee; Advisory Boards; Multiple Pulmonary Sites Workgroup. The IASLC Lung Cancer Staging Project: background data and proposed criteria to distinguish separate primary lung cancers from metastatic foci in patients with two lung tumors in the forthcoming eighth edition of the TNM classification for lung cancer. J Thorac Oncol. 2016;11: 651-65.

11. Detterbeck F, Marom EM, Arenberg D, Franklin WA, Nicholson AG, Travis WD et al; IASLC Staging and Prognostic Factors Committee; Advisory Boards; Multiple Pulmonary Sites Workgroup. The IASLC Lung Cancer Staging Project: background data and proposals for the application of TNM staging rules to lung cancer presenting as multiple nodules with ground glass or lepidic features or a pneumonic-type of involvement in the forthcoming eighth edition of the TNM classification. J Thorac Oncol. 2016;11:666-80.

12. Travis D, Asamura H, Bankier AA, Beasley MB, Detterbeck F, Flieder DB, et al; International Association for the Study of Lung Cancer Staging and Prognostic Factors Committee and Advisory Board Members. The IASLC Lung Cancer Staging Project: proposals for coding $\mathrm{T}$ categories for subsolid nodules and assessment of tumor size in part-solid tumors in the forthcoming eighth edition of the TNM classification of lung cancer. J Thorac Oncol. 2016;11:1204-23.

13. Detterbeck F. Developing a prognostic prediction model for lung cancer. In: Rami-Porta R, ed. Staging Manual in Thoracic Oncology. 2nd ed. North Fort Meyers (FL): Editorial Rx Press; 2016:237-58. 